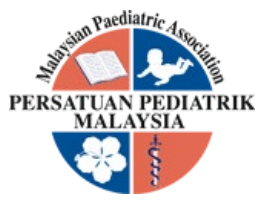

\title{
PAEDIATRIC EXTENSIVE CERVICAL PLEXIFORM NEUROFIBROMA MIMICKING A PAROTID LESION
}

\author{
Lim Iu Tong, , Avatar Singh Mohan Singh², Viji Ramasamy2, Suhana Abdul Rahim², \\ Ang Piao Piao ${ }^{3}$, Irfan Mohamad"
}

\begin{abstract}
Neurofibromatosis Type 1 (NF1), also known as von Recklinghausen's disease is a multisystem genetic disorder that occurs with an incidence of 1 in 4000 live births. Plexiform nmeurofibroma (PN) is a rare important variant of NF1, seen in 5 to $15 \%$ of cases. We report a 7 -year-old boy, without family history of neurofibromatosis presented with left neck swelling associated with multiple café au lait spots for one-year duration. Computed tomography of head and neck revealed an extensive soft tissue lesion involving the left parapharyngeal, carotid space and parotid space encasing the left internal and common carotid artery until its origin at the arch of aorta with erosion of the basiocciput. He underwent transcervical excision of the lesion, which was confirmed histopathologically as PN.
\end{abstract}

Keywords: Neurofibromatosis, Plexiform Neurofibroma, Malignant Peripheral Nerve Sheath Tumour

\section{Introduction}

Neurofibromatosis Type 1 (NF1), also known as von Recklinghausen's disease, is a neurocutaneous syndrome first described in 1882 by Friedrich von Recklinghausen [1]. NF1 is a multisystem genetic disorder that occurs with an incidence of 1 in 4000 live births [2]. It is inherited in an autosomal dominant form with irregular penetrance and variable expressivity with the gene locus located on chromosome 17, which encodes for tumour suppressor protein, called neurofibromin. However up to $50 \%$ of cases of NF1 have new mutation, meaning each child of a parent with NF1 runs a possibility $50 \%$ risk of carrying the disease [3]. Neurofibromin is the protein product of NF1 gene, which functions as a tumour suppressor protein expressed in many types of cells, predominantly glial cells, neuron, and Schwann cells that surround nerves. It assists in the regulation of cell proliferation and differentiation via activating GTPase activity and
Received: 08 September 2020; Accepted revised manuscript: 19 October 2020

Published online: 02 December 2020 subsequently inhibits Ras function and tyrosine kinase activity [3]. Due to the mutation and defect in this tumour suppressor gene, affected individuals are at risk of developing a variety of benign and malignant nervous system tumours, most notably benign neurofibromas.

Histologic subtypes of neurofibromas including the subcutaneous, cutaneous, nodular and diffuse

'Department of Otorhinolaryngology, Head and Neck Surgery, School of Medical Sciences, Universiti Sains Malaysia, 16150 Kubang Kerian, Kelantan, Malaysia ${ }^{2}$ Department of Otorhinolaryngology-Head and Neck Surgery, Hospital Taiping, 34000, Perak, Malaysia ${ }^{3}$ Department of Pathology, Hospital Taiping, 34000, Perak, Malaysia

Corresponding author: Professor Dr. Irfan Bin Mohamad, Department of OtorhinolaryngologyHead and Neck Surgery, School of Medical Sciences, Universiti Sains Malaysia, 16150 Kubang Kerian, Kelantan, Malaysia. Tel: +609-7676420

Email: irfankb@usm.my 
plexiform variants [4]. Plexiform neurofibroma (PN) is rare and seen in only 5 to $15 \%$ of cases with NF1 [5]. Histopathologically, PN is a subtype of benign nerve sheath tumour, characterized by proliferation of Schwann cells in the nerve sheath extending across the length of a nerve, and involving multiple fascicles and multiple nerves. Grossly, these convoluted masses are diffuse and infiltrative giving a clinical impression of 'bag of worms' appearance [6, 7].

Extra space before PN is a rare variant of NF1. In this article, we have the opportunity to share a rare case of extensive cervical PN in a 7-year-old boy with NF1, and discuss regarding the management of this difficult-to-manage disease.

\section{Case report}

A 7-year-old boy with underlying glucose-6phosphate dehydrogenase deficiency presented with a one-year history of painless nonprogressive left neck swelling. Patient had no fever, change of voice, noisy breathing, aspiration, or feeding difficulty. There was no history of trauma over the neck region. The child was born from non-consanguineous marriage, with normal birth and developmental history. There was no significant family history of neurofibromatosis.

General physical examination showed a moderately nourished child with no signs of stridor. On neck examination, there were multiple firm masses over the left infra-auricular region, level Ib, II and V cervical region, with largest $3 \mathrm{~cm} x$ $3 \mathrm{~cm}$ in diameter. The masses were non-pulsatile and non-tender with no superficial vessels, pigmentation, discharge, or ulceration seen. The masses were well demarcated and no bruit heard. Interestingly, patient had multiple wellcircumscribed, dark brown pigmentation on the skin (more than 6 in number and greater than 15 $\mathrm{mm}$ in diameter) over the abdomen and the back, demonstrated as segmental café au lait macules and patches. No pathognomonic features of NF1 such as axillary and inguinal freckling were present. The child have normal gait and there were no kyphosis or scoliosis deformity and limb length discrepancy to suggest skeletal system involvement in NF1. Vision and hearing of the child were clinically normal. Other systemic examinations were within normal limits. Routine haematological tests were within normal limits.
There was no clinical evidence of inflammatory and autoimmune disease.

As parent claimed the neck swelling was slightly reduced after the first course of oral antibiotic given by general practitioner prior to our first visit, the empirical treatment was continued for another course. Provisional diagnosis during the first visit was parotitis with cervical lymphadenopathy. Despite patient received a total of two courses of oral antibiotics, but swellings were persistent. Ultrasonography of neck showed features of ongoing inflammation of the left parotid gland with cervical lymphadenopathy. Fine needle aspiration for cytology (FNAC) was attempted during early assessment but procedure was abandoned as child was not cooperative. He underwent incisional biopsy of left level II cervical mass. The histopathological study report showed reactive lymphadenopathy with no evidence of malignancy. No mycobacterial tuberculosis was isolated or detected from the acid fast bacilli smear and culture result. He was closely observed as no malignancy was assured from the biopsy.

In view of the swelling did not resolve despite completing two courses of antibiotics and observation for 5 months, a contrasted computed tomography (CT) of head and neck was obtained. It revealed an extensive soft tissue lesion involving the left parapharyngeal, carotid space and parotid space (Figure 1). Medially, it extended to involve the prevertebral space and causing medialization and narrowing of airway. Posteriorly, it involved the posterior cervical space and perivertebral space. Inferiorly, it extended to encase the left internal carotid artery, common carotid artery until its origin at the arch of aorta. Superiorly, there was widening of the left petro-occipital fissure and foramen lacerum. Erosion of the basiocciput with focal lytic lesion seen at left parietal bone.

In view of the progression of the lesion and anticipating airway compromise, a transcervical excision was arranged. Intraoperatively, dissection was extraordinarily tedious because of the microvascularity of the tumour and loss of identifiable tissue planes. Mass was noted at left level 2 and involved tail of parotid region, arising from the parapharyngeal space (Figure 2). The bulk about $80 \%$ of the mass was resected, with residual lesion persisting in the parapharyngeal space, because the complete removal of this extensive mass in the neck was impossible and significant bleeding was encountered during the excision. 

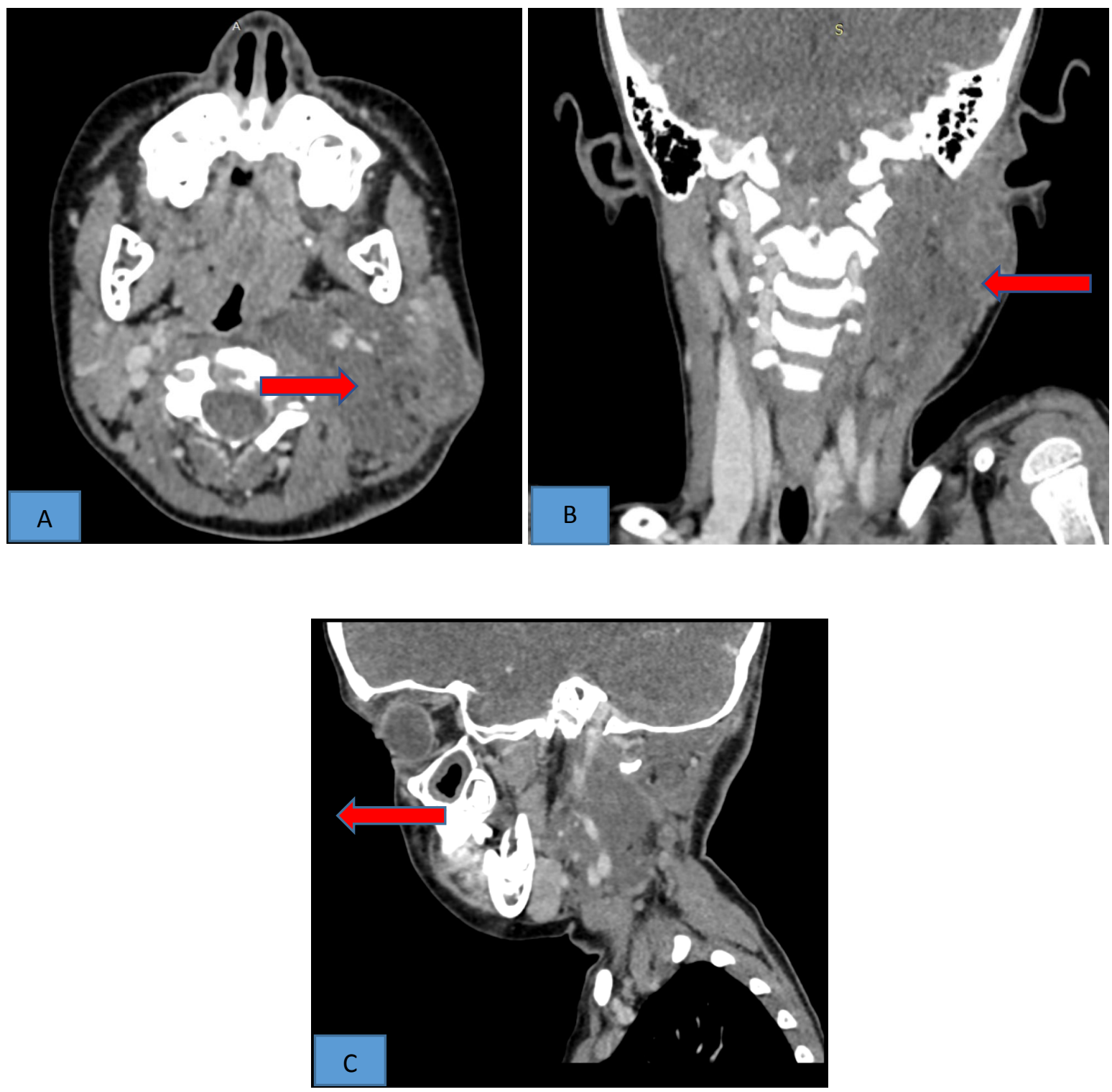

Figure 1. CT revealed extensive soft tissue lesion involving the left parapharyngeal, carotid space and parotid space as visualized in axial view (A), coronal view (B), and sagittal view (C). 

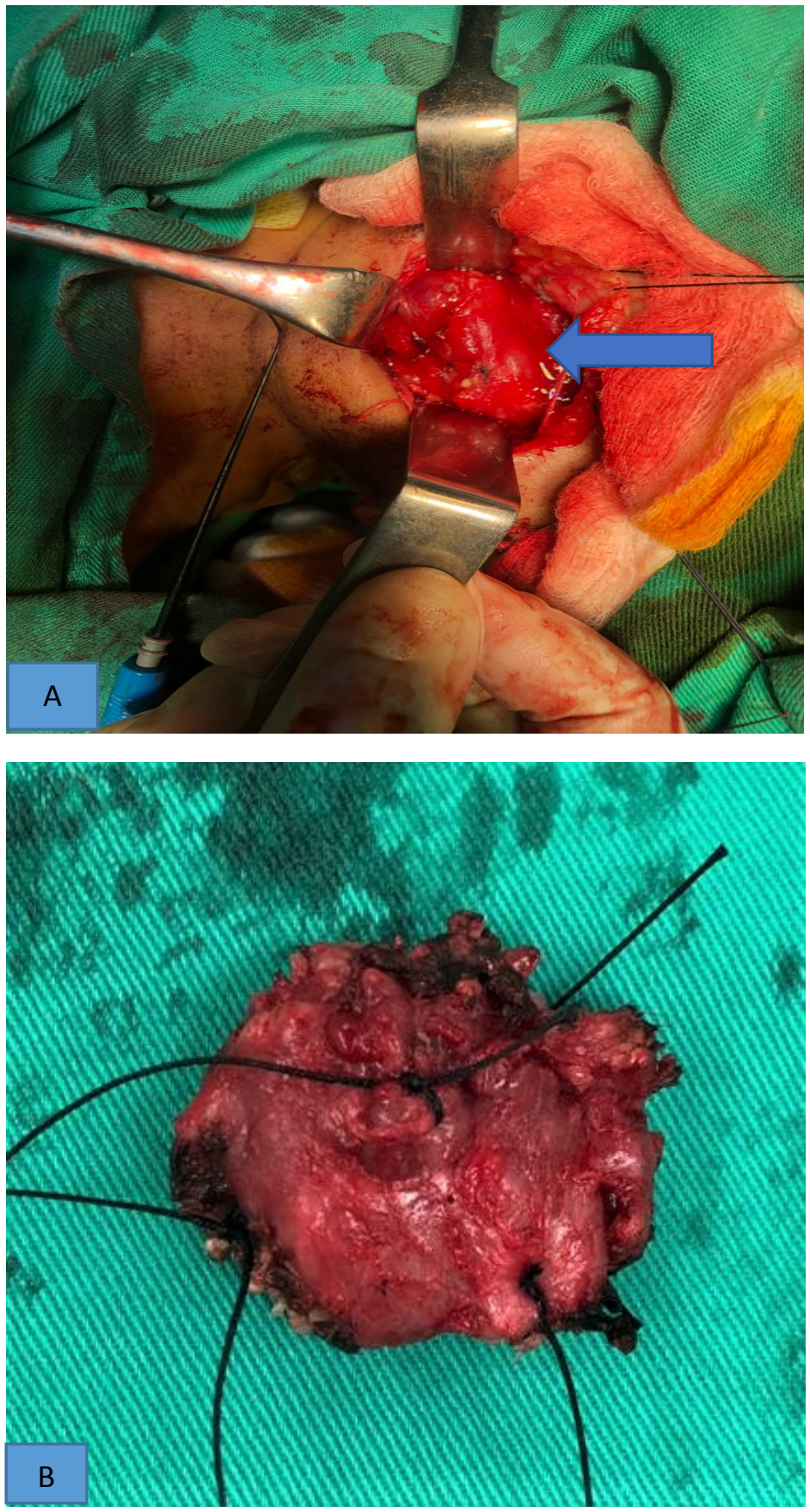

Figure 2. Clinical photograph showing the bulk of the cervical mass observed intra-operatively $(A)$ and the specimen resected $(B)$.

On Day 2 post operation, patient developed painful haematoma over the operation site. Patient was sent to operation theatre for wound exploration. Multiple bleeding spots were noted over the wound bed, which were controlled with diathermy and compression. Subsequently, he recovered well and was discharged. Histopathological examination revealed an ill- defined tumour infiltrating parotid tissue splaying the secretory units composed of multiple, irregularly arranged structures resembling hypertrophic nerve fascicles. The nerve fascicles were expanded by dispersed tumour cells enmeshed in abundant myxoid matrix, composed of spindle-shaped cells with tapered nuclei and waxy eosinophilic cytoplasm. No evidence of 
malignancy seen. The tumour cells were positive for the immunohistochemical staining with $\mathrm{S} 100$ protein. These features were highly suggestive the diagnosis of PN (Figure 3). Until now, patient was followed up for 12 months with no recurrence or new symptoms.
The patient was then referred to ophthalmologist and pediatrician to rule out other features of NF1 and exclude the possibility of any other systemic involvement. No iris hamartomas (Lisch nodules) or astrocytomas were found on slit lamp examination in both eyes. Dilated fundus evaluation and intraocular pressure were within normal limits.
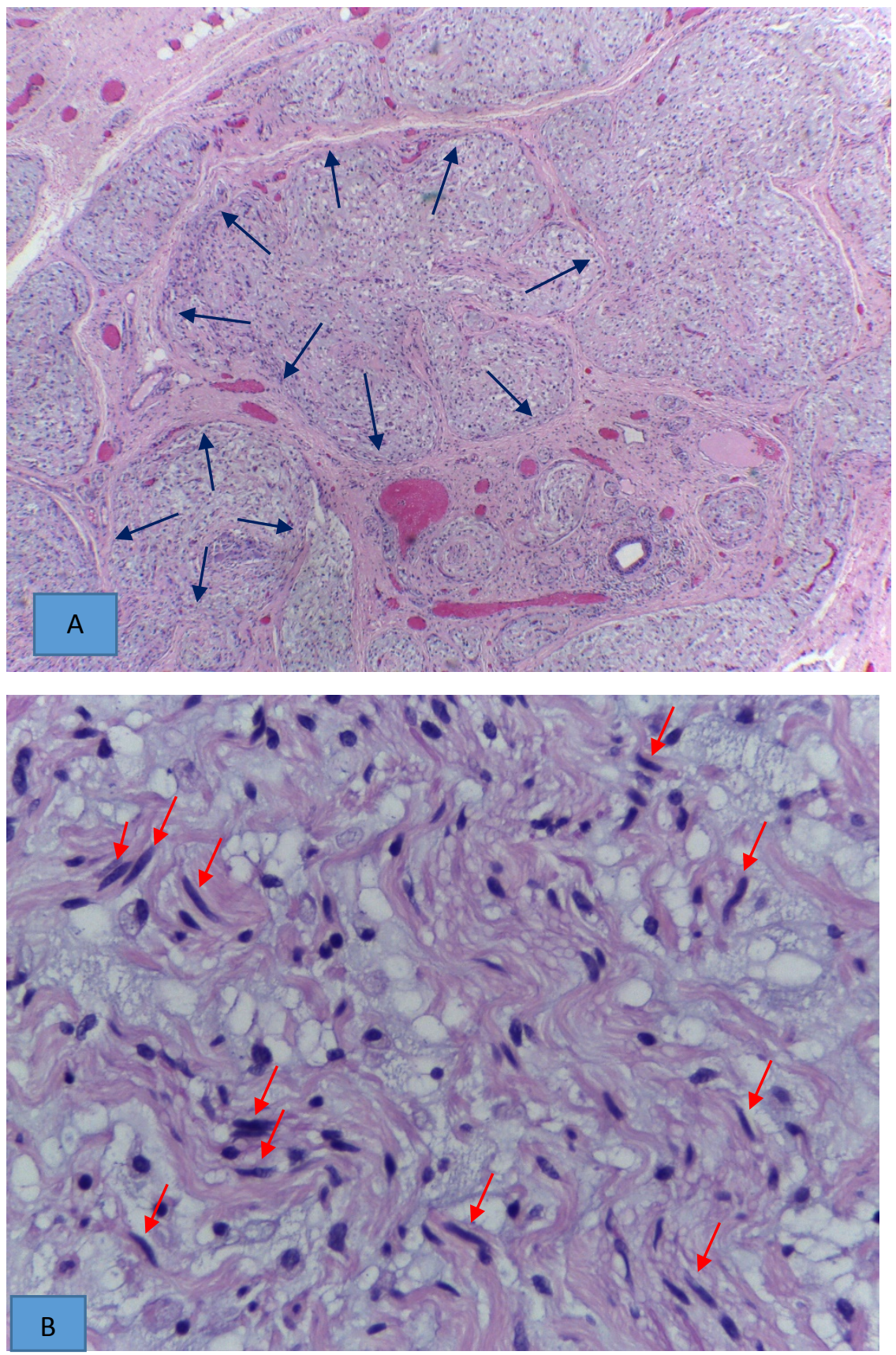

Figure 3. Histopathology of PN showing an ill-defined tumour infiltrating parotid tissue splaying the secretory units composed of multiple, irregularly arranged structures resembling hypertrophic nerve fascicles (A). The nerve fascicles were expanded by dispersed tumour cells enmeshed in abundant myxoid matrix, composed of spindle-shaped cells with tapered nuclei and waxy eosinophilic cytoplasm (B). 


\section{Discussion}

PN are typically congenital, non-metastatic, intraneural tumour that occur approximately 50\% in head and neck, facial and larynx region [8]. PN found in $30 \%$ cases of NF1, its local invasive feature may result in cosmetic deformities and functional damage [7]. Due to their potential of growth and ability to occupy space, massive PN can cause a mass effect and compression toward the adjacent important head and neck structures, including airway, spinal cord and orbit and lead to impairment of neurological structures [8, 9]. Moreover, they can cause soft tissue hyperplasia and resorption of underlying bone. In our case, medialization and narrowing of airway with erosion of the basiocciput were observed in the imaging.

Even though genetic testing is available, NF1 is usually diagnosed clinically based on the 1987 National Institute of Health criteria, which identified the NF1 in patients with two or more of the following findings: 1) Six or more café-au-lait macules over $5 \mathrm{~mm}$ in diameter in prepubertal individuals and over $15 \mathrm{~mm}$ in greatest diameter in postpubertal individuals, 2) Two or more neurofibromas of any type or one plexiform neurofibroma, 3) Freckling in the axillary or inguinal regions, 4) Two or more Lisch nodules (iris hamartomas), 5) Optic glioma, 6) A distinctive osseous lesion such as sphenoid dysplasia or thinning of long bone cortex, with or without pseudarthrosis, 7) First degree relative with NF1 (10). Our case had multiple (more than 6) café au lait spots of size $>5 \mathrm{~mm}$ with the diagnosis of cervical PN which confirmed by histopathological examination and thus fulfilled the diagnostic criteria of NF1.

Extra space PN characteristically benign, it has the potential for malignant transformation. Unlike the other variants, PN carries 3 to $4 \%$ risk of change into malignant peripheral nerve sheath tumour (MPNST), which carries extremely poor prognosis [11]. The risk of malignancy transformation in PN can achieved 10\% [12]. Once diagnosed as MPNST, patients have a 5-year survival rate of 20 to $50 \%$ only. Prognosis for MPNST is usually poor due to firstly, the diagnosis is difficult because it often present as a small focus within a large benign $\mathrm{PN}$, making incisional biopsy an unreliable method of detection as experienced in this case report. Secondly, once diagnosed, wide surgical excision is the only reliable treatment, and there were lack of evidence to support effective adjuvant therapies [13]. Upon discharge, our patient was given regular follow up to look for progression of disease or malignant transformation.

The diagnosis of cervical PN is an uncommon event. During the initial assessment of paediatric neck swelling while overlooked the features of NF1, misdiagnosis and mistreatment could be happened. Thus this report intended to raise the awareness regarding the possibility of extensive cervical PN and it should always be considered in cases of neck swelling with the presence of café au lait spots over the body to prevent delay in diagnosis and mistreatment.

Nowadays, the mainstay management of PN is still complete surgical resection. No specific treatment for PN currently exists, other than surgical resection $[14,15]$. However, success of surgical management of cervical PN in paediatric patients is limited and challenging due to the infiltrating nature of the tumour to surrounding tissues, massive growth and tendency for regrowth [16]. In our case, during resection of this bulky cervical mass, complete resection was impossible and we encountered intratumoural haemorrhage and it was complicated with post-operative haemorrhage where haemostasis was secured under general anesthesia.

Due to the invasive nature of $\mathrm{PN}$, these masses are usually non-resectable and their recurrence rate can be up to $20 \%$ even with surgical excision at best effort [14]. The major problem during intraoperation is difficulty to separate the tumour from normal tissue because they are non-encapsulated tumour which consisting of a mesh of connective tissues, axon and spindle cells [17]. Extensive lesion may be associated with massive haemorrhage and excessive bleeding in PN during operation can be due to the friable vasculature secondary to vascular invasion by tumour itself or arterial dysplasia [18]. PN is not radiosensitive plus the consideration of the risk of radiotherapy inducing malignant transformation in benign PN, radiotherapy option is not recommended. Other options such as chemotherapy have only very limited benefits to patients due to PN's slow growth rate [8].

Although FNAC is a fast, simple, and minimally invasive technique, it was not reliable to give accurate diagnosis in certain infiltrative parotid lesions. There are high levels of deficient in 
diagnosis and missed malignancies with high nondiagnostic rates average $14-18 \%$ and can be as high as $56 \%$. Therefore, it may affect the outcome of diagnosis and definitely the treatment. Moreover, even when FNAC is optimized, cellular aspirate cannot be utilized for grading, staging or immunofluorescence, thus contributes to difficulty in interpretation [19]. Despite open incisional biopsy is invasive and have its disadvantages, it still generally considered as gold standard for obtaining a final diagnosis and most of the times provide adequate sample for achieving a diagnosis [20]. Interestingly, open biopsy is no longer justified as high risk in tumour seeding, facial nerve injury, and fistula formation in some major salivary gland tumour [21].

\section{Conclusion}

Head and neck NF1 is a rare clinical entity, but attending surgeon should always considering cervical PN as one of the differential diagnosis of neck mass in children because it is critical for early diagnosis and treatment procedure. Diagnosis of NF1 is established when patients fulfilled two or more criteria as stated by National Institute of Health. Small cervical PN can be surgically removed if diagnosed in early childhood, and eliminating the possibility of airway compression, cosmetic deformity and neurological deficits. Surgical intervention is the mainstay of treatment for PN and MPNST. The indication and timing of surgical intervention in pediatric patients must be carefully evaluated against the functional disturbances and psychological consequences of surgery. Further intervention such as radiotherapy and chemotherapy are usually not required. This case report highlights the importance of early diagnosis of PN, its surgical difficulty and possible complications.

\section{References}

[1] Ruggieri M. The different forms of neurofibromatosis. Childs Nerv Sys 1999;15:295-308.

[2] Crawford AH, Schorry EK. Neurofibromatosis in children: the role of the orthopaedist. J Am Acad Orthop Surg. 1999;7:217-30.

[3] Upadhyaya M, Cooper DN, eds. Neurofibromatosis Type 1. Berlin: SpringerVerlag; 2012: 187-209.
[4] Hartley N, Rajesh A, Verma R, Sinha R, Sandrasegaran K. Abdominal manifestations of neurofibromatosis. J Comput Assist Tomogr. 2008;32(1):4-8.

[5] Mobashir MK, Mohamed AE, El-Anwar MW, El Sayed $A E$, Fouad MA. Massive plexiform neurofibroma of the neck and larynx. Int Arch Otorhinolaryngol. 2015;19(4):349-53.

[6] Enzinger FM, Weiss SW. Soft tissue tumors. $2^{\text {nd }}$ ed. St Louis: CV Mosby. 1988. 745-56.

[7] Kleihues P, Louis DN, Scheithauer BW, Rorke LB, Reifenberger G, Burger PC, et al. The WHO classification of tumors of the nervous system. J Neuropathol Exp Neurol. 2002;61:215-25.

[8] Jeffrey BW, Jonathan EC, Jean BB, Ian J, Lisa E. Management of head and neck plexiform neurofibromas in pediatric patients with neurofibromatosis type 1. Arch Otolaryngol Head Neck Surg. 2005;131:712-8.

[9] Kerry PL, Edward PB, Joseph SG. Neurofibromatosis of the head and neck: classification and surgical management. Plast Reconstr Surg. 2015;135(3):845-55.

[10] National Institutes of Health Consensus Development Conferences Steatment: Neurofibromatosis. Bethesda, Md, USA, July 13-15,1987. Neurofibromatosis. 1988;1:172-8.

[11] Sabatini C, Milani D, Menni F, Tadini G, Esposito S. Treatment of neurofibromatosis type 1. Curr Treat Options Neurol 2015;17(6): 355.

[12] Jayachandran D, Sunantha S, Gopalaiah H, Veeraraghavan G. Plexiform neurofibromatosis involving face and oral cavity. J Oral Maxillofac Pathol. 2014;18(1):114-7.

[13] Jeffrey BW, Snehal GP, Jatin PS. Management issues in massive paediatric facial plexiform neurofibroma with neurofibromatosis type 1. Head Neck. 2002;24(2):207-11.

[14] Asha'ari ZA, Kahairi A, Shahid H. Surgery for massive paediatric head and neck neurofibroma: two case reports. The International Medical Journal Malaysia. 2012;11(2):54-7.

[15] Ransom ER, Yoon C, Manolidis S. Single stage near total resection of massive pediatric head and neck plexiform neurofibromas. Int J Pediatr Otorhinolaryngol. 2006;70(6):1055-61.

[16] Friedrich $R E$, Schmelzle $R$, Hartmann $M$, Mautner VF. Subtotal and total resection of superficial plexiform neurofibromas of face 
and neck: four case reports. J Craniomaxillofac Surg. 2005;33(1):55-60.

[17] Liu J, Wong CF, Lim F, Kanagalingam J. Glottic neurofibroma in an elderly patient: a case report. J Voice. 2013;27(5):644-6.

[18] Rao V, Affifi RA, Ghazarian D. Massive subcutaneous hemorrhage in a chest-wall neurofibroma. Can J Surg. 2000;43:459-60.

[19] Sananda H, Joseph DS, Kemal MT, Samuel ST, David CH. Biopsy of parotid masses: Review of current techniques. World J radiol. 2016;8 (5):501-5.

[20] Pfeiffer J, Kayser G, Technau-Ihling K, Boedeker CC, Ridder GJ. Ultrasound-guided core-needle biopsy in the diagnosis of head and neck masses: Indications, technique, and results. Head Neck. 2007;29(11):1033-40.

[21] Howlett DC. Diagnosing a parotid lump: fine needle aspiration cytology or core biopsy. British Journal of Radiology. 2006;79 (940):295-7. 